\title{
PBCA-based polymeric microbubbles for molecular imaging and drug delivery
}

Citation for published version (APA):

Koczera, P., Appold, L., Shi, Y., Liu, M., Dasgupta, A., Pathak, V., Ojha, T., Fokong, S., Wu, Z., van Zandvoort, M., Iranzo, O., Kuehne, A. J. C., Pich, A., Kiessling, F., \& Lammers, T. (2017). PBCA-based polymeric microbubbles for molecular imaging and drug delivery. Journal of Controlled Release, 259, 128135. https://doi.org/10.1016/j.jconrel.2017.03.006

Document status and date:

Published: 10/08/2017

DOI:

10.1016/j.jconrel.2017.03.006

Document Version:

Publisher's PDF, also known as Version of record

Document license:

Taverne

Please check the document version of this publication:

- A submitted manuscript is the version of the article upon submission and before peer-review. There can be important differences between the submitted version and the official published version of record.

People interested in the research are advised to contact the author for the final version of the publication, or visit the DOI to the publisher's website.

- The final author version and the galley proof are versions of the publication after peer review.

- The final published version features the final layout of the paper including the volume, issue and page numbers.

Link to publication

\footnotetext{
General rights rights.

- You may freely distribute the URL identifying the publication in the public portal. please follow below link for the End User Agreement:

www.umlib.nl/taverne-license

Take down policy

If you believe that this document breaches copyright please contact us at:

repository@maastrichtuniversity.nl

providing details and we will investigate your claim.
}

Copyright and moral rights for the publications made accessible in the public portal are retained by the authors and/or other copyright owners and it is a condition of accessing publications that users recognise and abide by the legal requirements associated with these

- Users may download and print one copy of any publication from the public portal for the purpose of private study or research.

- You may not further distribute the material or use it for any profit-making activity or commercial gain

If the publication is distributed under the terms of Article $25 \mathrm{fa}$ of the Dutch Copyright Act, indicated by the "Taverne" license above, 


\title{
PBCA-based polymeric microbubbles for molecular imaging and drug delivery
}

\author{
Patrick Koczera a,b,1, Lia Appold ${ }^{\mathrm{a}, 1}$, Yang Shi ${ }^{\mathrm{a}}$, Mengjiao Liu ${ }^{\mathrm{a}}$, Anshuman Dasgupta ${ }^{\mathrm{a}}$, Vertika Pathak ${ }^{\mathrm{a}}$, \\ Tarun Ojha a,h , Stanley Fokong a , Zhuojun $\mathrm{Wu}^{\mathrm{c}}$, Marc van Zandvoort ${ }^{\mathrm{c}, \mathrm{d}}$, Olga Iranzo ${ }^{\mathrm{e}}$, Alexander J.C. Kuehne ${ }^{\mathrm{f}}$, \\ Andrij Pich ${ }^{\mathrm{f}}$, Fabian Kiessling ${ }^{\mathrm{a}, *}$, Twan Lammers ${ }^{\mathrm{a}, \mathrm{g}, \mathrm{h}, *}$ \\ a Department of Experimental Molecular Imaging (ExMI), Helmholtz Institute for Biomedical Engineering, University Hospital RWTH, Pauwelsstr. 30,52074 Aachen, Germany \\ ${ }^{\mathrm{b}}$ Department of Intensive Care, University Hospital RWTH Aachen, Pauwelsstr. 30, 52074 Aachen, Germany \\ c Institute for Molecular Cardiovascular Research, University Hospital RWTH Aachen, Pauwelsstr. 30, 52074 Aachen, Germany \\ d Department of Genetics and Cell Biology - Molecular Cell Biology, Cardiovascular Research Institute Maastricht, Maastricht University Medical Centre, 6229 HX Maastricht, The Netherlands \\ e Aix Marseille Univ, CNRS, Centrale Marseille, iSm2, Marseille, France \\ ${ }^{\mathrm{f}}$ DWI - Leibniz Institute for Interactive Materials, RWTH Aachen University, Forckenbeckstr. 50, 52056 Aachen, Germany \\ ${ }^{g}$ Department of Targeted Therapeutics, Institute for Biomedical Technology and Technical Medicine, University of Twente, PO Box 217, 7500 AE Enschede, The Netherlands \\ ${ }^{\mathrm{h}}$ Department of Pharmaceutics, Utrecht Institute for Pharmaceutical Sciences, Utrecht University, Universiteitsweg 99, 3583 CG Utrecht, The Netherlands
}

\section{A R T I C L E I N F O}

\section{Article history:}

Received 23 January 2017

Received in revised form 23 February 2017

Accepted 3 March 2017

Available online 6 March 2017

\section{Keywords:}

Microbubbles

Ultrasound

Sonoporation

Nanomedicine

Tumor targeting

\begin{abstract}
A B S T R A C T
Microbubbles (MB) are routinely used as contrast agents for ultrasound (US) imaging. We describe different types of targeted and drug-loaded poly(n-butyl cyanoacrylate) (PBCA) MB, and demonstrate their suitability for multiple biomedical applications, including molecular US imaging and US-mediated drug delivery. Molecular imaging of angiogenic tumor blood vessels and inflamed atherosclerotic endothelium is performed by modifying the surface of PBCA MB with peptides and antibodies recognizing E-selectin and VCAM-1. Stable and inertial cavitation of PBCA MB enables sonoporation and permeabilization of blood vessels in tumors and in the brain, which can be employed for direct and indirect drug delivery. Direct drug delivery is based on US-induced release of (model) drug molecules from the MB shell. Indirect drug delivery refers to US- and MB-mediated enhancement of extravasation and penetration of co-administered drugs and drug delivery systems. These findings are in line with recently reported pioneering proof-of-principle studies showing the usefulness of (phospholipid) MB for molecular US imaging and sonoporation-enhanced drug delivery in patients. They aim to exemplify the potential and the broad applicability of combining MB with US to improve disease diagnosis and therapy.
\end{abstract}

(c) 2017 Elsevier B.V. All rights reserved.

\section{Introduction}

Ultrasound (US) imaging is, besides X-ray radiography, the most widespread clinical imaging modality. It combines the advantages of bedside availability and real-time imaging with cost- and timeeffectiveness [1]. MB in the range of 1-10 $\mu \mathrm{m}$, which consist of a gasor air-filled core stabilized by a lipid- or polymer-based shell, are routinely used as contrast agents for US imaging. In general, imaging of soft-shell lipid-based MB is done using non-destructive US and it is based on their non-linear oscillation upon exposure to low- to medium-intensity US pulses (stable cavitation); on the other hand, destructive pulses (inertial cavitation) and backscattering - which

\footnotetext{
* Corresponding authors.

E-mail address: tlammers@ukaachen.de (T. Lammers).

1 These authors contributed equally to this study
}

becomes more important at higher US frequencies - are more frequently employed in case of hard-shell polymer-based MB [2-4].

Due to their size, which is too large for extravasation, MB can only be employed as intravascular contrast agents, allowing e.g. for the assessment of the perfusion of small vessels, which cannot be properly visualized using contrast agent-free (e.g. Power-Doppler-based) US imaging [5]. This is used in clinical practice for diagnosis, staging and functional characterization of e.g. cardiovascular and hepatic pathologies [6,7]. Additionally, it can be used to characterize potential cancerous lesions, as angiogenesis is an important step in the transformation to malignancies, and as MB-based functional and molecular US imaging allow for accurate profiling, staging and treatment monitoring [6-8]. In such setups, the functional aspect of vessel imaging is restricted to the visualization and quantification of blood flow and flow velocity, while at the molecular level, information can be obtained on the (over-) expression of surface receptors, aiding in the more detailed characterization of tumor angiogenesis [9,11]. Among the surface receptors routinely used in 
molecular US imaging are classic angiogenic targets, like the vascular endothelial growth factor receptor 2 (VEGFR-2) and $\alpha_{\nu} \beta_{3}$ integrins, as well as inflammatory markers, such as the intercellular or vascular cell adhesion molecules ICAM and VCAM, and E- and P-selectins [10-14].

Besides for imaging, $\mathrm{MB}$ can also be used for drug delivery. The combination of MB and US can - via sonoporation and sonopermeabilization mechanisms - affect the integrity of the vascular lining and of cellular membranes [15-18]. This temporary perforation and the enhanced endo/transcytosis induced by stable and/or inertial MB cavitation is highly useful for temporally and spatially guiding drug delivery, e.g. to solid tumors or across the blood-brain barrier (BBB) [19-23]. The exact mechanisms contributing to enhanced drug delivery upon sonoporation are incompletely understood, as are differences in the sonoporation-potential of soft- vs. hard-shell $\mathrm{MB}$, and targeted vs. untargeted MB $[24,25]$. When exposed to US, MB rapidly shrink and swell, in line with the applied US frequency, and this results in physical effects such as microstreaming, microjets, ultrasonic shockwaves and $\mathrm{MB}$ compression/rarefaction-induced disruption of endothelial linings and cellular membranes [15-18,26,27]. These phenomena can contribute to enhanced extravasation, penetration and cellular uptake of drugs and drug delivery systems [20,21,28-30].

MB-mediated drug delivery can be subdivided into indirect and direct drug delivery. In case of the former, drugs or drug delivery systems are co-administered with MB and US. In case of direct drug delivery, drug molecules are embedded within the MB shell (or bound, in the form of drug delivery systems, to their surface) and locally released at the pathological site in a temporally and spatially controllable manner [31-33]. Such externally triggerable drug targeting concepts are increasingly employed, also in patients, as exemplified e.g. by efforts to combine US-mediated hyperthermia with temperature-sensitive liposomes in liver cancer [34-37], as well as by recently published pioneering advances showing that sonoporation can be favorably employed in patients with pancreatic cancer and glioblastoma $[38,39]$.

With regard to direct drug delivery, one could argue that polymeric $\mathrm{MB}$, because of their much thicker shell (50-150 nm), are advantageous as compared to lipid-based $\mathrm{MB}(3-5 \mathrm{~nm})$, providing more space for drug loading $[40,41]$. Polymeric MB might also allow for more diverse (and tailorable) modes of surface functionalization, via direct and indirect coupling mechanisms. In this manuscript, we exemplify the broad applicability of prototypic poly ( $n$-butyl cyanoacrylate) (PBCA)-based MB for molecular US imaging and drug delivery applications. We extend previously published concepts for targeted US imaging of atherosclerosis and tumor angiogenesis, and for direct and indirect US-mediated drug delivery to tumors and to the brain. These findings exemplify the usefulness of combining MB with US for more efficient disease diagnosis and therapy.

\section{Materials and methods}

\subsection{Synthesis of PBCA-based polymeric microbubbles}

PBCA MB were synthesized by adding $3 \mathrm{ml}$ of $n$-butyl cyanoacrylate (BCA, Special Polymers, Sofia, Bulgaria) dropwise to $300 \mathrm{ml}$ of an aqueous solution containing $1 \%$ of Triton X-100 (Sigma Aldrich, Munich, Germany) at pH 2.5. The solution was stirred for $1 \mathrm{~h}$ at $10,000 \mathrm{rpm}$ and room temperature, and a suspension of air-filled polydispersed $\mathrm{MB}$ was obtained. MB with defined sizes were separated using multiple centrifugation and flotation steps. Additional washing steps were performed to purify the MB dispersion. MB size and concentration were measured with a Beckman Coulter Multisizer 3. Electron microscopy was performed using a Hitachi S4800 FESEM for scanning electron microscopy (SEM) with a Cryo-Gatan unit Alto 2500 at $1 \mathrm{kV}$ and $2 \mu \mathrm{A}$. A drop of the aqueous MB suspension was placed on the sample holder and frozen using liquid nitrogen, before insertion into the preparation chamber. A Philips EM400T was used for transmission electron microscopy (TEM). The samples were fixed using $2 \%$ agarose and dehydrated with ethanol before embedding in liquid epoxy resin. The resins were cut into $85 \mathrm{~nm}$ sections.

\subsection{Drug loading into the shell of $P B C A M B$}

The loading of (model) drug molecules into the shell of PBCA-based polymeric MB was performed via a one-step or two-step approach. Fluorescent dyes can be employed as model drugs, allowing for simple and straightforward quantification of loading and release, and facilitating in vitro and in vivo evaluation of cellular internalization and target site accumulation. In the one-step approach, $2.5 \mathrm{mg}$ of rhodamine B (AppliChem, Darmstadt, Germany) and/or coumarin 6 (Sigma Aldrich, Munich, Germany) were dissolved in $300 \mu \mathrm{H}_{2} \mathrm{O}$ or dimethylformamide (DMF, Carl Roth, Karlsruhe, Germany), and added at once before the $\mathrm{BCA}$ to the polymerization medium during the MB formation process.

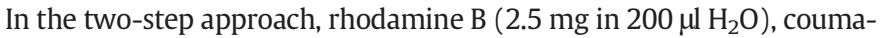
rin 6 ( $2.5 \mathrm{mg}$ in $200 \mu \mathrm{LMF}$ ) and/or the near infrared fluorophore $1,1^{\prime}, 3,3,3^{\prime}, 3^{\prime}$ hexamethyl-indotricarbocyanine-iodide (HITC, Sigma Aldrich, Munich, Germany) (10 mg in $100 \mu$ DMSO (Carl Roth, Karlsruhe, Germany)) were post-loaded into the MB shell. Unloaded dye is removed from the MB-solution via several washing steps. MB were allowed to float and the solution underneath was refreshed until no free dye was left. After initially preparing a batch of size-optimized, surface-functionalized and/or actively targeted MB, the model drug solutions were added to $10 \mathrm{ml}$ prepared $\mathrm{MB}$ under continuous stirring at room temperature. Measurements of fluorophore-loaded MB were performed using a Leica TCS SP8 X automated inverted confocal and stimulated emission depletion (STED) microscope equipped with a planapochromat $100 \times / 1.40$ oil-immersion objective.

\subsection{Surface functionalization and modification with targeting ligands}

MB-shell functionalization was done via the EDC (i.e. $\mathrm{N}$-(3dimethylaminopropyl)- $\mathrm{N}$-ethylcarbodiimide hydrochloride, Sigma Aldrich, Munich, Germany) coupling chemistry. The butyl-ester groups of the PBCA side-chains were partly hydrolyzed by increasing the $\mathrm{pH}$ to around 10 with $\mathrm{NaOH}$ ( $0.1 \mathrm{M}$, AppliChem, Darmstadt, Germany) for $15 \mathrm{~min}$. The resulting carboxyl groups were activated by EDC $\left(5 \times 10^{8} \mathrm{MB}, 7.5 \mathrm{mg} \mathrm{EDC}\right)$ and streptavidin $(300 \mu \mathrm{g}$, ChemImpex, Wood Dale, USA) or the E-selectin binding peptide GGG-IELLQAR ( $1 \mathrm{mg}$, Olga Iranzo, Marseille, France) were added to the MB suspension followed by stirring at $4{ }^{\circ} \mathrm{C}$ overnight. The functionalized MB were purified by several washing steps to remove the unreacted EDC and peptides and size-separated by centrifugation and flotation. Streptavidinlabeled MB $\left(1 \times 10^{7}\right)$ were further functionalized by coupling to biotinylated antibodies directed against VCAM-1 (5 $\mu$ g, clone 429, eBioscience, Frankfurt, Germany) and VEGFR-2 (0.75 $\mu \mathrm{g}$, Abcam, Cambridge, UK).

\subsection{Ex vivo studies}

All in vivo and ex vivo experiments were approved by institutional and governmental ethics boards. Fluorescent rhodamine B-loaded MB targeted against VCAM-1 via antibodies were used in 8-week-old male CD-1 wild-type mice. Endothelial activation (mimicking vascular inflammation) was achieved by intraperitoneal injection of recombinant murine TNF- $\alpha$ (PeproTech, Hamburg, Germany). Carotid arteries were excised $4 \mathrm{~h}$ after endothelial activation and mounted in a home-built flow-chamber. After 10 min of infusion with VCAM-1-targeted MB or unmodified control MB (diluted to a final concentration of $10^{7} \mathrm{MB} / \mathrm{ml}$ in full blood; at a flow rate of $0.25 \mathrm{ml} / \mathrm{min}$ ), carotid arteries were imaged ex vivo by two-photon laser scanning microscopy using an Olympus FV1000MPE multiphoton microscopy system (Mai Tai DeepSee pulsed Ti:Sapphire laser with $140 \mathrm{fs}$ pulse width at an excitation wavelength of $800 \mathrm{~nm}$ ) with a $25 \times$ water dipping objective. 


\subsection{In vivo studies}

To visualize and characterize tumor angiogenesis, E-selectintargeted and non-targeted control $\mathrm{MB}\left(1 \times 10^{7} \mathrm{MB}\right)$ were i.v. injected in CD1 nude mice bearing subcutaneous A431 human epidermoid cancer xenografts (ATCC CRL-1555; 6 mice per group). Replenishment analysis was used to distinguish between bound and unbound MB. US imaging was performed in the non-linear contrast mode using a VisualSonics Vevo2100 preclinical ultrasound system with a MS250 transducer operating at a frequency of $18 \mathrm{MHz}$ of the tumor xenografts up until $7 \mathrm{~min}$ after i.v. injection. Before and after a destructive pulse, the mean contrast intensity in the tumor region was compared. This was done after the replenishment phase, and allows for accurate monitoring of the expression of vascular markers, such as E-selectin. To test the ability of the PBCA MB for direct drug delivery purposes, mice with s.c. CT26 (ATCC CRL-2638) colon carcinoma allografts were injected with coumarin 6-loaded anti-VEGFR-2 antibody-targeted MB. MB were destroyed in the tumor vasculature by multiple destructive US pulses (non-linear contrast mode, Vevo2100, $18 \mathrm{MHz}, \mathrm{MI}=1$ ) at 7 min after MB injection, when the majority of the MB fraction in tumors corresponded to vesselassociated US contrast agents. Vessels were stained with i.v. injected rhodamine-labeled lectin at the end of the experiment, and tumor sections were used for histology to image MB-mediated delivery, accumulation and penetration of coumarin 6 in mice with CT26 using fluorescence microscopy.

We also evaluated indirect enhancement of drug delivery, via sonoporation of the BBB. This was done in healthy CD1 nude mice, using $8.5 \times 10^{8}$ USPIO-loaded MB i.v. (infused for $5 \mathrm{~min}$ into the tail vein) in combination with co-injected FITC-labeled dextran (70 kDa, Sigma Aldrich, Munich, Germany; $200 \mathrm{mg} / \mathrm{kg}$ in $0.9 \%$ saline). Power Doppler US was performed for $5 \mathrm{~min}$ at a frequency of $16 \mathrm{MHz}$ over the skull of mice compared to control animals, which only received MB without US. Perfused blood vessels were stained using rhodaminelabeled lectin, enabling simultaneous microscopy imaging of the vasculature and of FITC-dextran extravasation and penetration. Tumors and brain tissue were harvested, mounted, frozen and cut using a Leica CM3050S cryostat. Tissue sections $(8 \mu \mathrm{m})$ were analyzed using the Carl Zeiss Axiolmager M2 microscopy system.

\section{Results and discussion}

\subsection{Preparation and functionalization of PBCA-based polymeric microbubbles}

The synthesis of PBCA MB, and representative scanning and transmission electron microscopy images are shown in Fig. 1A. Chemical modification of the shell of PBCA MB allows for functionalization of their surface, which facilitates specific binding of the MB (Fig. 1B). After partial hydrolysis of the MB shell, surface functionalization was performed via EDC-coupling with streptavidin and subsequent use of biotinylated antibodies, as well as via direct EDC coupling of small oligomeric peptides (Fig. 1C). The specificity of the targeted MB was initially tested in vitro. To this end, the binding of targeted MB to activated endothelial cells was compared, by means of fluorescent microscopy, to non-targeted MB as well as to targeted MB binding under blocking conditions $[42,43]$. Results were confirmed in vivo (see below). Using such setups, multiple antibody-targeted PBCAbased MB have already been shown to hold potential for molecular US imaging [42,44-46].

The straightforward synthesis of PBCA MB leads to MB dispersions with relatively broad size distribution. After 1-3 centrifugation/flotation rounds, a $\mathrm{MB}$ population with an acceptable size distribution and with a mean size of $\sim 2 \mu \mathrm{m}$ was isolated (Fig. 2A). Optimized centrifugation/flotation protocols furthermore allow for the isolation of MB batches with varying size populations, i.e. 1, 2 and $3 \mu \mathrm{m}$ in diameter (Fig. 2D). Afterwards, hydrophilic and hydrophobic model drugs (i.e. rhodamine B, coumarin 6 and HITC) were loaded into the shell of PBCA MB (Fig. 2BC). Successful co-loading of rhodamine $B$ and coumarin 6 was also demonstrated (right panel in Fig. 2C). Cryo-scanning electron microscopy was employed as a means to assess the shell-thickness of PBCA MB. In Fig. 2F-G, MB were destroyed and cryogenically fixed, to visualize the outer and inner structure of the shell. In these SEM analyses, the shellthickness appeared to be in the order of $50 \mathrm{~nm}$. STED microscopy (of intact MB) on the other hand indicated a shell-thickness of 150-200 nm (Fig. 2E). This difference in shell thickness likely results from the shrinkage of the MB shell during the freezing process required for cryo-SEM. It is anticipated that the results obtained using STED microscopy more accurately reflect the real shell thickness of PBCA MB.

These results exemplify the versatility of PBCA MB for targeting and shell loading, and they demonstrate that they can be easily synthesized and customized for purposes ranging from molecular imaging to drug delivery.

\section{2. $P B C A M B$ for molecular imaging in angiology}

We developed VCAM-1-targeted MB by coupling anti-VCAM-1 antibodies to the shell of rhodamine B-loaded PBCA MB via biotinstreptavidin linkages. VCAM-1 is known to be overexpressed on endothelial cells affected by inflammatory stimuli, e.g. in case of atherosclerosis. This is schematically depicted in Fig. 3A. In line with this, Fig. 3B shows the ex vivo binding of VCAM-1-targeted PBCA MB to the inflamed endothelial lining of TNF- $\alpha$-activated carotid arteries by 3D two-photon laser scanning microscopy (3D-TPLSM) in comparison to non-targeted MB. These ex vivo flow-chamber results are in line with the in vivo data previously reported by Curaj et al., who showed that VCAM-1-targeted MB are useful for longitudinally monitoring vascular healing after arterial injury [47]. They longitudinally examined carotid arteries of ApoE deficient mice under high fat diet in vivo and ex vivo by VCAM-1-targeted CEUS and 3D-TPLSM after wire-injury. The VCAM-1-specific PBCA MB showed accumulation at the injured site and variation of enhancement in the course of regeneration, with a peak after 3 days.

A related target for molecular US imaging of atherosclerosis is ICAM-1. In a similar flow-chamber setup, TNF- $\alpha$ induced arterial inflammation resulted in the retention of ICAM-1-targeted MB in the carotid artery. This was analyzed ex vivo both by US imaging and by microscopy, and findings were validated in vivo. With this approach, $\mathrm{Wu}$ and colleagues earlier already showed specific lesionretention of ICAM-1-targeted MB, even under high (arterial) shear stress conditions, making this molecular US imaging approach potentially useful for differentiating between stable and vulnerable atherosclerotic plaques [48]. Similarly, Rix and colleagues recently tested $\alpha_{v} \beta_{3}$ integrin-targeted MB after vascular injury in pigs. In this study, employing MB modified with cyclic RGD peptide as a targeting moiety, it was found that $\alpha_{\mathrm{v}} \beta_{3}$ integrin-targeted MB bind to endoarterial balloon injury sites, with a peak in integrin expression and MB binding after 1 week, and normalization back to baseline levels after 3 months [49].

The usefulness of antibody-targeted PBCA MB was also evaluated in neuroinflammation. ICAM-1 and VCAM- 1 targeting was realized by coupling the respective biotinylated antibodies to streptavidin-coated MB. Contrast-enhanced US detected specific localization of these MB in vivo and ex vivo in the brain and spinal cord of rats with adoptive transfer experimental autoimmune encephalomyelitis; responses to anti-inflammatory corticosteroid therapy could also be assessed, as the accumulation of ICAM-1-targeted MB decreased upon drug treatment [50,51].

Together, these findings show that PBCA MB represent a versatile platform for molecular imaging of vascular injury and inflammation in angiology, addressing endothelial biomarkers such as VCAM-1, ICAM1 and $\alpha_{\mathrm{v}} \beta_{3}$ integrins. 
A

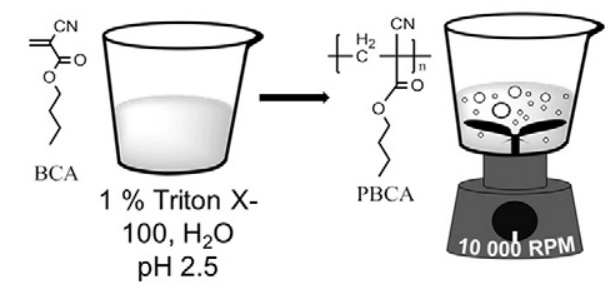

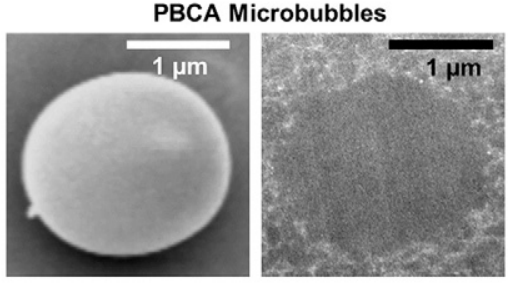

B
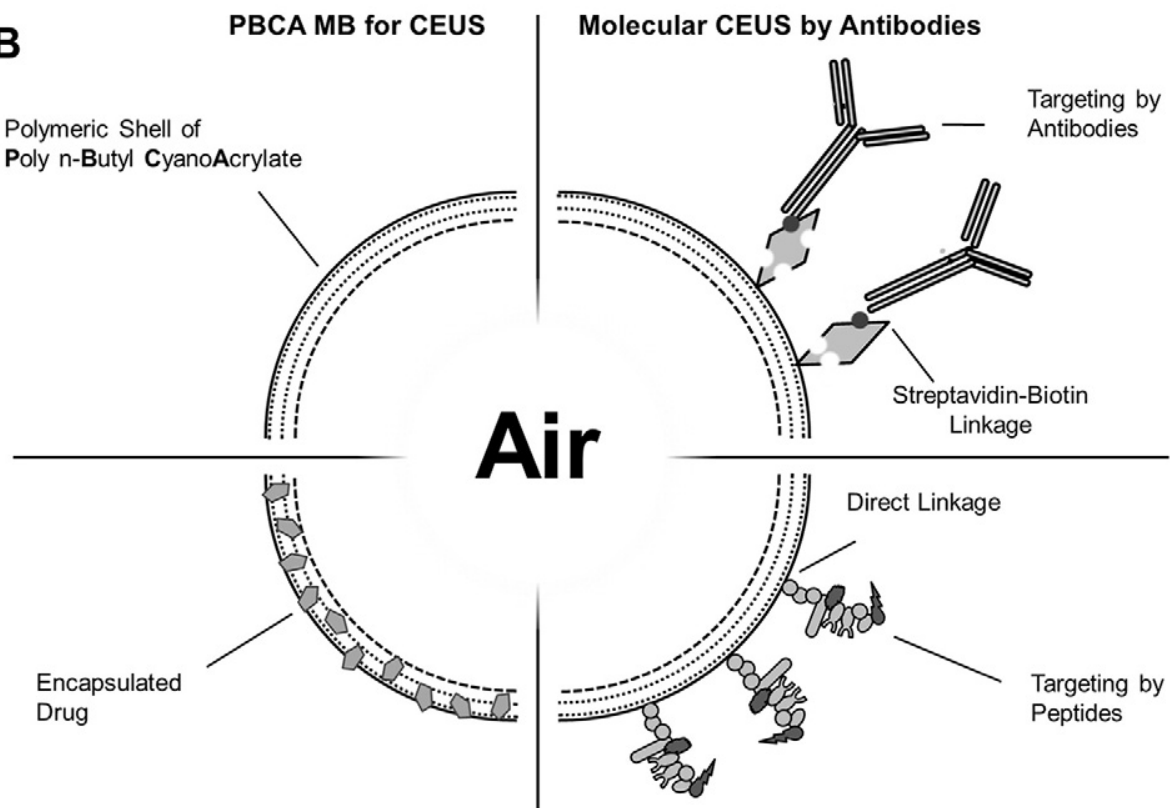

MB for Direct Drug Delivery

Molecular CEUS by Peptides

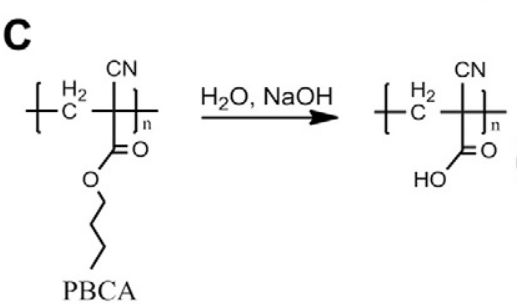

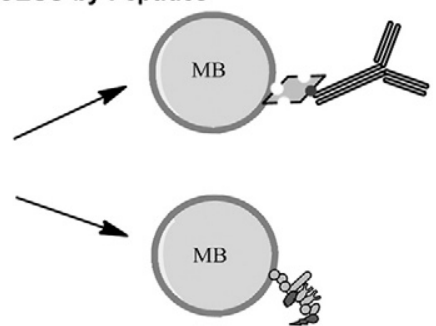

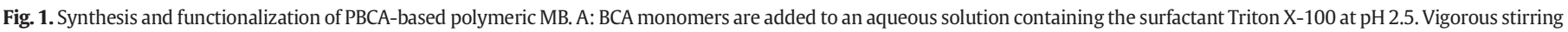

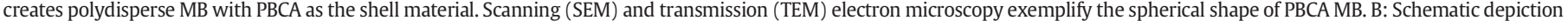

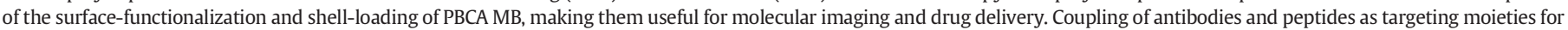

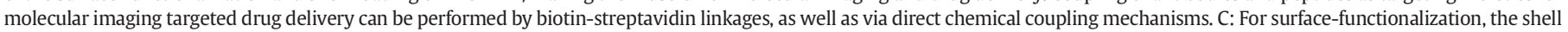
of PBCA MB is hydrolyzed to create carboxyl-groups for chemical coupling to targeting ligands.

\subsection{PBCA MB for molecular imaging in oncology}

Antibody- and peptide-targeted MB also hold potential for molecular US imaging in oncology (Fig. 3C). To extend efforts in this direction, we coupled the IELLQAR peptide to the MB to target E-selectin, which is a marker for angiogenesis and which is known to be overexpressed during tumor progression. In A431 squamous cell carcinoma xenografts, these MB showed specific accumulation: when comparing pre- and post-MB US images, clear enhancements in signal intensity were observed (Fig. 3D). The difference in signal intensity represents MB bound to the vascular wall, and is a measure for angiogenic marker expression. These findings in A431 tumors confirm previously published findings with E-selectin-targeted MB in MLS human ovarian cancer xenografts [46]. Spivak et al. used the same E-selectin-targeted MB to assess the impact of $\mathrm{MB}$ dosing. A dose of $7 \times 10^{7} \mathrm{MB} / \mathrm{kg}$ body weight was found to be sufficient to detect E-selectin expression in tumors and to monitor the effect of antiangiogenic therapy [52].
Another prominent target for molecular US imaging in oncology is VEGFR-2. Fokong and colleagues developed VEGFR-2-targeted PBCA MB by biotin/streptavidin-based antibody coupling, and showed specific accumulation and molecular US imaging in subcutaneous CT26 colon carcinoma xenografts in mice [53]. Along the same line of thinking, Palmowski et al. used VEGFR-2-targeted PBCA MB, and also $\alpha_{v} \beta_{3}$ integrin- and ICAM1-targeted MB, for specific CEUS imaging and for the evaluation of changes in angiogenic biomarkers during anti-matrix-metalloproteinase therapy [54]. Moreover, in a syngeneic rat prostate carcinoma model, they observed specific accumulation of ICAM-1- and $\alpha_{\mathrm{v}} \beta_{3}$-integrin-targeted $\mathrm{MB}$, and an increase in both biomarkers upon heavy-ion radiotherapy (as opposed to a decrease in vessel density and vascular perfusion; which was found using functional US imaging) [55].

These preclinical examples illustrate the utility of targeted PBCA MB for molecular imaging and treatment monitoring of tumors. Relevant endothelial markers like VEGFR-2, E-selectin, ICAM-1, VCAM-1 and $\alpha_{v} \beta_{3}$-integrin can be specifically addressed via modifying the surface 

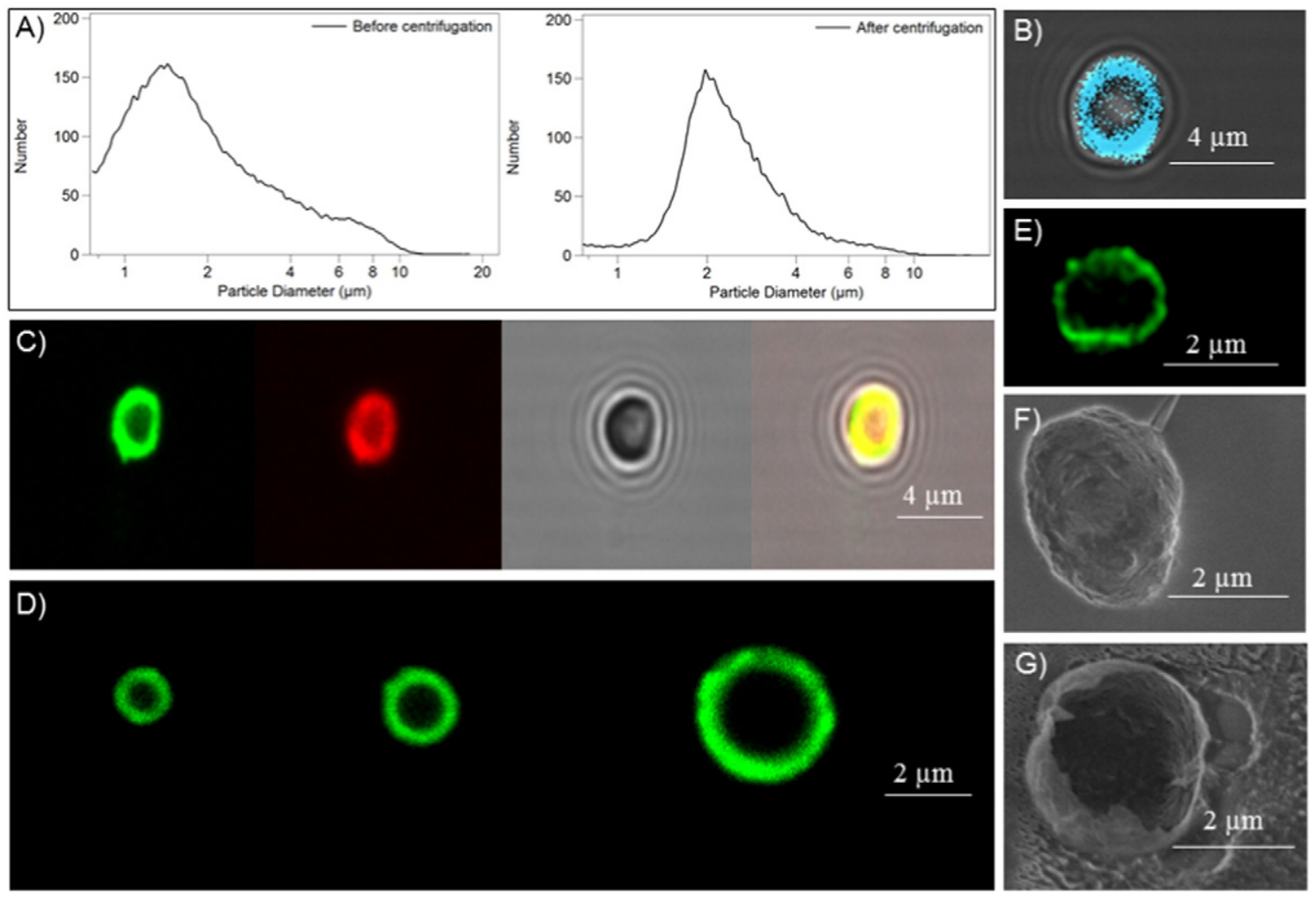

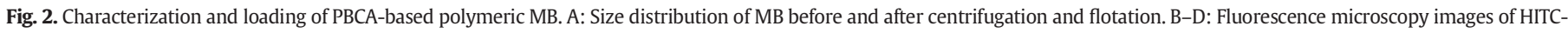

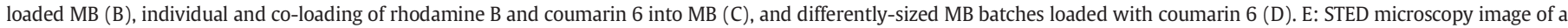
coumarin 6-loaded MB. F-G: Scanning electron microscopy (SEM) images of an intact (F) and a destroyed (G) PBCA MB.

of PBCA MB with antibodies and peptides. The clinical evaluation of such targeted US contrast agents has recently been initiated, albeit with phospholipid-based MB, looking e.g. at the expression levels of VEGFR-2 in prostate cancer patients [56,57].

\subsection{PBCA MB for indirect drug delivery}

Indirect drug delivery refers to sonoporation, which can assist in the permeation of endothelial linings and/or cell membranes. We analyzed the accumulation of the $70 \mathrm{kDa}(10 \mathrm{~nm})$ macromolecular model drug FITC-dextran in brain tissue after co-injection with PBCA MB and US treatment, and compared it with the accumulation and penetration of FITC-dextran in non-sonicated controls (Fig. 4A-B). Fluorescence microscopy showed high accumulation of the FITC-dextran around blood vessels in the brains of mice receiving MB and US treatment. No signal of extravasated FITC-dextran was visible in the brains of mice which were injected with MB but not treated by US, exemplifying successful indirect drug delivery across the $\mathrm{BBB}$ and to the brain upon sonoporation [58].

We mirrored these experiments in solid tumors, looking at the accumulation and penetration of $100 \mathrm{~nm}$-sized fluorophore-labeled PEGylated liposomes in tumor models with low baseline levels of enhanced permeability and retention (EPR), i.e. highly cellularly dense A431 epidermoid xenografts and highly stromal BxPC3 pancreatic xenografts [59]. We compared PBCA-based polymeric $\mathrm{MB}$ to phospholipid-based $\mathrm{MB}$, but did not observe obvious differences. It is worth mentioning, however, that the sonoporation protocols (dosing of MB and US, US setting, timing, etc.) have not yet been optimized to allow for accurate/detailed assessment of differences in sonoporation potential. Another interesting question is whether vascular targeting (e.g. using antibodies or peptides targeted to VEGFR-2 or E-selectin) enhances the efficacy of sonoporation. As sonoporation potential likely directly depends on the distance of oscillating/destroyed MB to the endothelial lining, targeting may allow for more efficient (and safe; in case of the brain) sonoporation. Finally, it has to be mentioned in this context that MB- and US-mediated indirect drug delivery has recently been performed in patients, albeit with phospholipid-based MB. These pioneering studies have shown that prolonged survival times can be obtained in pancreatic cancer patients treated with gemcitabine upon combining it with sonoporation [38,56,57]. A first trial in glioblastoma patients, treated with doxorubicin in combination with sonoporation has also recently started, and the outcomes of this study are eagerly awaited [39].

Together, these efforts exemplify the potential of combining MB with US to induce sonoporation of blood vessels at pathological sites, promoting the targeted delivery of drugs and drug delivery systems to tumors and to the brain.

\subsection{PBCA MB for direct drug delivery}

Direct drug delivery refers to the use of MB containing drug molecules within their shell, and to their temporally and spatially controlled release upon exposure to US (Fig. 4C). To provide initial proof-ofprinciple for direct drug delivery using PBCA-based $\mathrm{MB}$, the fluorophores coumarin 6 (as a hydrophobic model drug) and rhodamine $\mathrm{B}$ (as a hydrophilic model drug) were encapsulated into the MB shell [53]. These MB were targeted to VEGFR-2 and their targetspecific accumulation was confirmed in subcutaneous CT26 colon carcinoma tumors. For the evaluation of direct (model) drug delivery to tumors, 2D fluorescence microscopy was employed. As hypothesized, and as shown in Fig. 4D (upper panel), the US-mediated destruction of PBCA MB in tumors resulted in efficient accumulation and penetration of the hydrophobic model drug coumarin 6. No accumulation was observed in control tumors, which were not exposed to US (lower panel). 


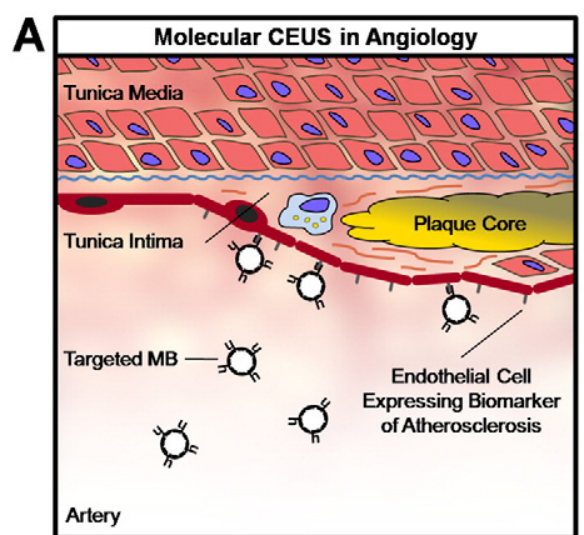

B

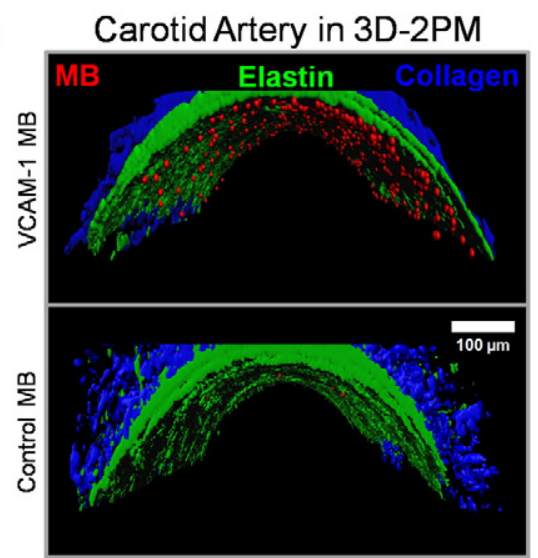

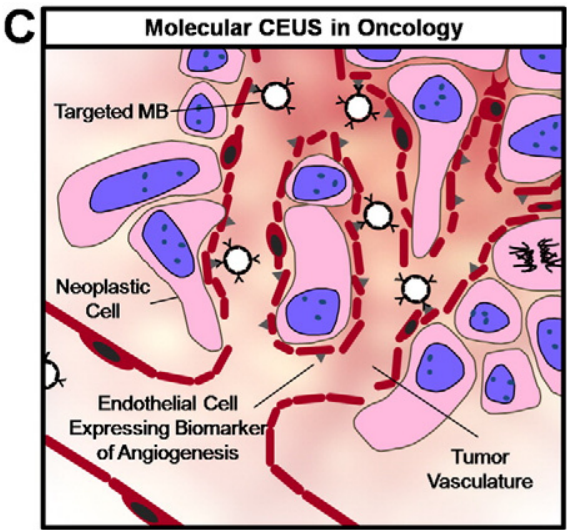

D

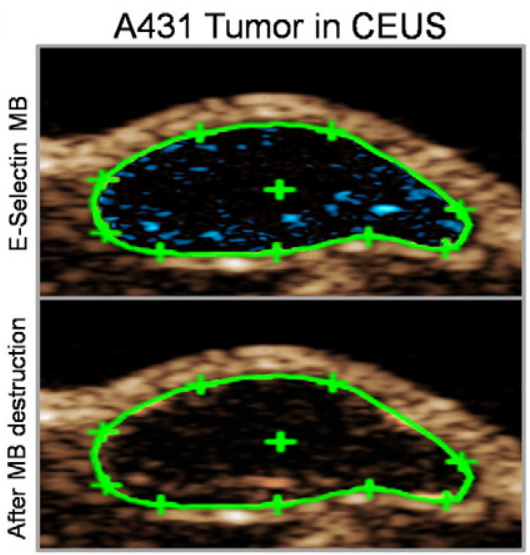

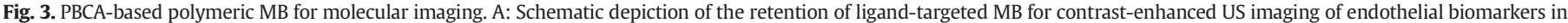

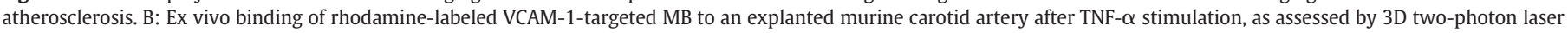

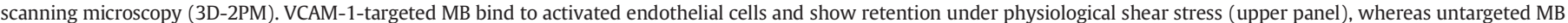

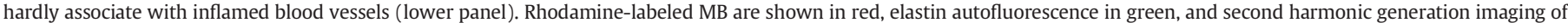

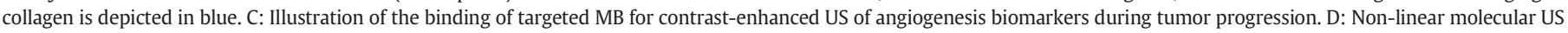

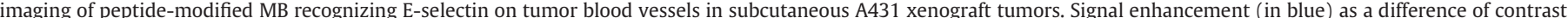

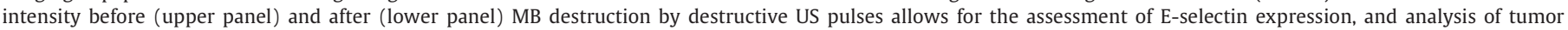
vascularization and angiogenesis.

In a similar setup, Wheatley and colleagues used polylactic acid (PLA)-based MB for direct drug delivery of doxorubicin and paclitaxel to tumors. In the VX2 liver tumor model in New Zealand rabbits, nontargeted doxorubicin-loaded PLA MB were locally destroyed by US at the tumorous site during circulation. ${ }^{14} \mathrm{C}$-labeling of doxorubicin enabled assessment of the target site accumulation of the drug. In comparison to non-sonicated tumors, significantly enhanced levels of doxorubicin were found in tumor tissue [60]. Extending these findings, in the Morris rat hepatocellular carcinoma model, doxorubicin-loaded MB induced tumor growth inhibition upon US-mediated MB destruction at the pathological site [61]. PLA MB loaded with paclitaxel have also been developed and employed. In in vitro model systems, based on the human breast cancer cell lines MDA-MB-231 and MCF7, these $\mathrm{MB}$ induced significantly more cell death when combined with US than when no US was applied [63].

In an alternative approach for MB-mediated direct drug delivery, pioneered (and patented) by scientists at SINTEF in Norway, the polymeric MB shell is composed of model drug-loaded PBCA nanoparticles (NP). Upon sonication, these MB disintegrate into 100-200 nm-sized nanoparticles, which can be deposited across sonoporated endothelium. Using these PBCA NP-stabilized MB, temporally and spatially controlled disruption of the BBB has already been demonstrated, as exemplified by the detection of Nile Red-loaded PBCA NP in the brain, as well as by the accumulation of the co-injected gadolinium-based MRI contrast agent Omniscan ${ }^{\mathrm{TM}}$ in sonicated regions [62]. Using the same setup, PBCA NP delivery to and into prostate adenocarcinoma xenografts upon US exposure has also already been demonstrated, as evidence by fluorescence microscopy detection [64].

These findings show that PBCA-based polymeric MB hold potential for direct drug delivery to tumors and to the brain, with drug molecules either embedded into the MB shell, or into NP constituting the MB shell, allowing for US-controlled delivery to pathological sites.

\section{Conclusion}

We demonstrate the broad applicability of PBCA-based polymeric $\mathrm{MB}$ for molecular imaging and drug delivery. Evidence is provided showing that (model) drug molecules can be efficiently loaded into the shell of PBCA MB. In addition, VCAM-1-targeting of inflamed blood vessels is shown in ex vivo flow chambers, and the feasibility of Eselectin-specific MB imaging of tumor angiogenesis is confirmed in A431 tumors. Furthermore, MB- and US-mediated sonoporation is demonstrated to result in BBB opening and in the accumulation of the macromolecular model drug FITC-dextran in the brain. Finally, we show that shell-incorporated model drugs can be efficiently shuttled across the vascular wall in CT26 tumors. These results exemplify the usefulness of PBCA MB for molecular US imaging of atherosclerosis and tumor angiogenesis. They also indicate that sonoporation-enhanced direct and indirect drug delivery to tumors and to the brain may contribute to more efficient disease treatment. Together, the findings summarized 


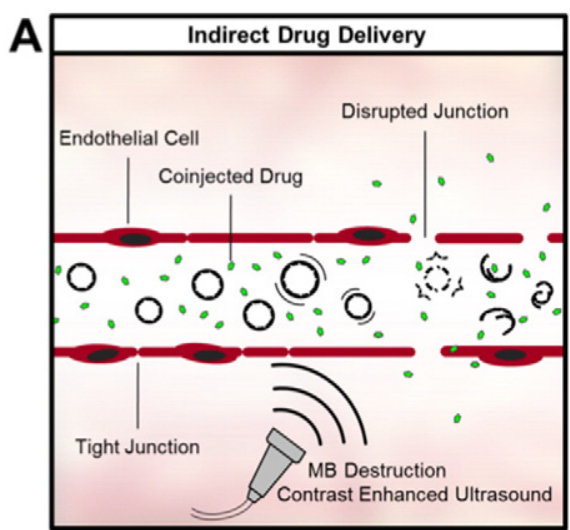

B

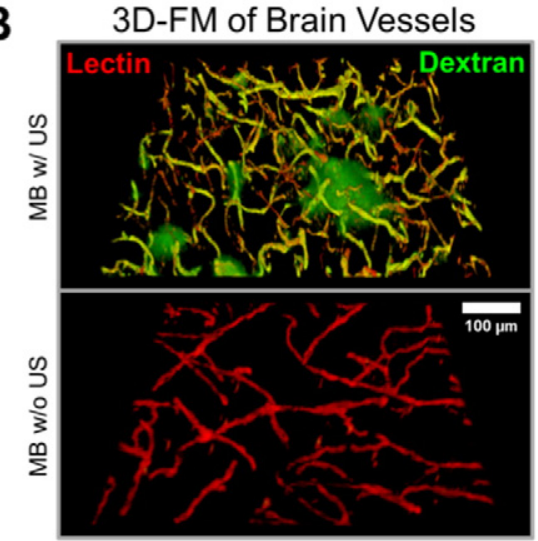

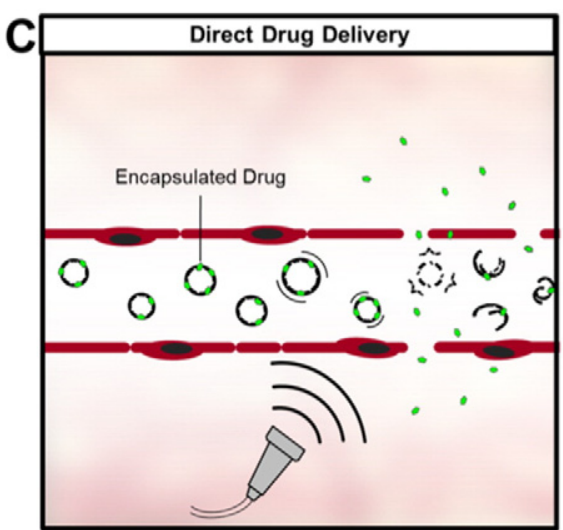

D 2D-FM of Tumor Vessels

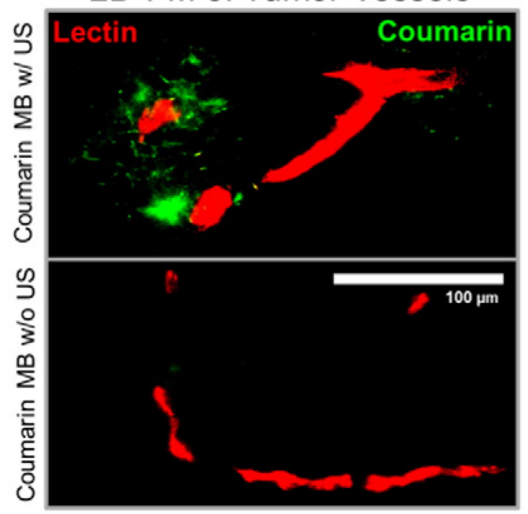

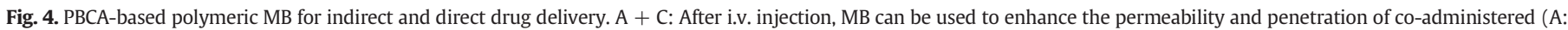

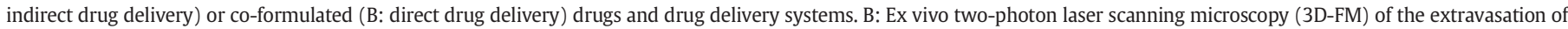

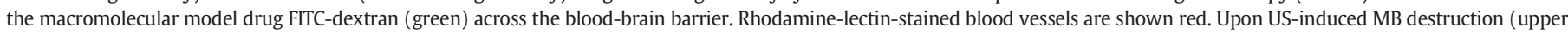

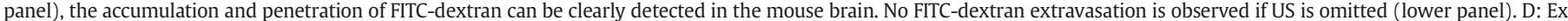

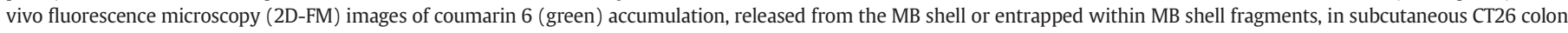

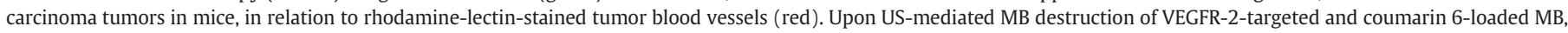
substantially enhanced model drug delivery to and into tumorous tissue can be observed.

in this study illustrate the potential and the broad applicability of PBCAbased polymeric MB.

\section{Acknowledgements}

The authors gratefully acknowledge financial support by the German Research Foundation (DFG: LA 2937/1-2), by the European Research Council (ERC-StG-309495-NeoNaNo), by the German Center for Cardiovascular Research (DZHK: FKZ 81X2800152), and by the $i^{3}$ tm Seed Fund Program (which is part of the Excellence Initiative of the German federal and State Governments; SF_14-4-09-Sonoporation). The authors also gratefully acknowledge input from the IZKF multiphoton imaging facility at RWTH Aachen University Clinic for assistance with the TPLSM experiments (Dr. Michael Vogt).

\section{References}

[1] P.N.T. Wells, Ultrasound imaging, Phys. Med. Biol. 51 (13) (2006) 83.

[2] A. Bauer, et al., Wideband harmonic imaging: a novel contrast ultrasound imaging technique, Eur. Radiol. 9 (3) (1999) 364-367.

[3] K. Tiemann, et al., Stimulated acoustic emission: pseudo-Doppler shifts seen during the destruction of nonmoving microbubbles, Ultrasound Med. Biol. 26 (7) (2000) 1161-1167.

[4] D. Cosgrove, Future prospects for SonoVue and CPS, Eur. Radiol. Suppl. 14 (8) (2004) 116-124.

[5] J. Ehling, T. Lammers, F. Kiessling, Non-invasive imaging for studying anti-angiogenic therapy effects, Thromb. Haemost. 109 (3) (2013) 375-390.

[6] D. Strobel, et al., Tumor-specific vascularization pattern of liver metastasis, hepatocellular carcinoma, hemangioma and focal nodular hyperplasia in the differential diagnosis of 1349 liver lesions in contrast-enhanced ultrasound (CEUS), Ultraschall Med. 30 (04) (2009) 376-382.
[7] S.L. Mulvagh, et al., American society of echocardiography consensus statement on the clinical applications of ultrasonic contrast agents in echocardiography, J. Am. Soc. Echocardiogr. 21 (11) (2008) 1179-1201.

[8] M. Palmowski, et al., Vessel fractions in tumor xenografts depicted by flow- or contrast-sensitive three-dimensional high-frequency Doppler ultrasound respond differently to antiangiogenic treatment, Cancer Res. 68 (17) (2008) 7042-7049.

[9] S.C. Baetke, et al., Squamous cell carcinoma xenografts: use of VEGFR2-targeted microbubbles for combined functional and molecular US to monitor antiangiogenic therapy effects, Radiology 278 (2) (2015) 430-440.

[10] D. Hanahan, R.A. Weinberg, Hallmarks of cancer: the next generation, Cell 144 (5) (2011) 646-674.

[11] F. Kiessling, S. Fokong, J. Bzyl, W. Lederle, M. Palmowski, T. Lammers, Recent advances in molecular, multimodal and theranostic ultrasound imaging, Adv. Drug Deliv. Rev. 72 (2014) 15-27.

[12] D.J. Hicklin, L.M. Ellis, Role of the vascular endothelial growth factor pathway in tumor growth and angiogenesis, J. Clin. Oncol. 23 (5) (2005) 1011-1027.

[13] P. Libby, P.M. Ridker, A. Maseri, Inflammation and atherosclerosis, Circulation 105 (9) (2002) 1135-1143.

[14] J.D. Hood, D.A. Cheresh, Role of integrins in cell invasion and migration, Nat. Rev Cancer 2 (2) (2002) 91-100.

[15] S.I. Grivennikov, F.R. Greten, M. Karin, Immunity, inflammation, and cancer, Cell 140 (6) (2010) 883-899.

[16] J.R. Lindner, J. Song, J. Christiansen, A.L. Klibanov, F. Xu, K. Ley, Ultrasound assessment of inflammation and renal tissue injury with microbubbles targeted to Pselectin, Circulation 104 (17) (2001) 2107-2112.

[17] R.R. Karshafian, P.D. Bevan, R. Williams, S. Samac, P.N. Burns, Sonoporation by ultrasound-activated microbubble contrast agents: effect of acoustic exposure parameters on cell membrane permeability and cell viability, Ultrasound Med. Biol. 35 (5) (2009) 847-860.

[18] T.A. Tran, J.Y.L. Guennec, P. Bougnoux, F. Tranquart, A. Bouakaz, Characterization of cell membrane response to ultrasound activated microbubbles, IEEE Trans. Ultrason. Ferroelectr. Freq. Control 55 (1) (2008) 43-49.

[19] M. Derieppe, K. Rojek, J.-M. Escoffre, B.D. de Senneville, C. Moonen, C. Bos, Recruitment of endocytosis in sonopermeabilization-mediated drug delivery: a real-time study, Phys. Biol. 12 (4) (2015) 046010.

[20] A. van Wamel, et al., Vibrating microbubbles poking individual cells: drug transfer into cells via sonoporation, J. Control. Release 112 (2) (2006) 149-155. 
[21] E.C. Unger, E. Hersh, M. Vannan, T.O. Matsunaga, T. McCreery, Local drug and gene delivery through microbubbles, Prog. Cardiovasc. Dis. 44 (1) (2001) 45-54.

[22] Y. Liu, H. Miyoshi, M. Nakamura, Encapsulated ultrasound microbubbles: therapeutic application in drug/gene delivery, J. Control. Release 114 (1) (2006) 89-99.

[23] I. Lentacker, S.C.D. Smedt, N.N. Sanders, Drug loaded microbubble design for ultrasound triggered delivery, Soft Matter 5 (11) (2009) 2161-2170.

[24] C.-Y. Ting, et al., Concurrent blood-brain barrier opening and local drug delivery using drug-carrying microbubbles and focused ultrasound for brain glioma treatment, Biomaterials 33 (2) (2012) 704-712.

[25] H.-L. Liu, C.-H. Fan, C.-Y. Ting, C.-K. Yeh, Combining microbubbles and ultrasound for drug delivery to brain tumors: current progress and overview, Theranostics 4 (4) (2014) 432-444

[26] J.R. Lindner, Microbubbles in medical imaging: current applications and future directions, Nat. Rev. Drug Discov. 3 (6) (2004) 527-533.

[27] F. Kiessling, S. Fokong, P. Koczera, W. Lederle, T. Lammers, Ultrasound microbubbles for molecular diagnosis, therapy, and theranostics, J. Nucl. Med. 53 (3) (2012) 345-348.

[28] I. Lentacker, I. De Cock, R. Deckers, S.C. De Smedt, C.T.W. Moonen, Understanding ultrasound induced sonoporation: definitions and underlying mechanisms, Adv. Drug Deliv. Rev. 72 (2014) 49-64.

[29] A. Dasgupta, M. Liu, T. Ojha, G. Storm, F. Kiessling, T. Lammers, Ultrasound-mediated drug delivery to the brain: principles, progress and prospects, Drug Discov. Today Technol. 20 (2016) 41-48.

[30] B.H.A. Lammertink, C. Bos, R. Deckers, G. Storm, C.T.W. Moonen, J.-M. Escoffre, Sonochemotherapy: from bench to bedside, Front. Pharmacol. 6 (2015) 138.

[31] M.R. Böhmer, C.H.T. Chlon, B.I. Raju, C.T. Chin, T. Shevchenko, A.L. Klibanov, Focused ultrasound and microbubbles for enhanced extravasation, J. Control. Release 148 (1) (2010) 18-24

[32] F.-Y. Yang Y.-S. Lin, K.-H. Kang, T.-K. Chao, Reversible blood-brain barrier disruption by repeated transcranial focused ultrasound allows enhanced extravasation, J. Control. Release 150 (1) (2011) 111-116.

[33] T. Lammers, F. Kiessling, W.E. Hennink, G. Storm, Drug targeting to tumors: principles, pitfalls and (pre-) clinical progress, J. Control Release 161 (2) (2012) 175-187.

[34] M.J. Ernsting, A. Worthington, J.P. May, T. Tagami, M.C. Kolios, S.D. Li, Ultrasound drug targeting to tumors with thermosensitive liposomes, IEEE Int. Ultrason. Symp. 1-4 (2011)

[35] R.M. Staruch, M. Ganguly, I.F. Tannock, K. Hynynen, R. Chopra, Enhanced drug delivery in rabbit VX2 tumours using thermosensitive liposomes and MRI-controlled focused ultrasound hyperthermia, Int. J. Hyperth. 28 (8) (2012) 776-787.

[36] A. Kheirolomoom, et al., Complete regression of local cancer using temperaturesensitive liposomes combined with ultrasound-mediated hyperthermia, J. Control. Release 172 (1) (2013) 266-273.

[37] Targeted chemotherapy using focused ultrasound for liver tumours (TARDOX), Clin Trials Gov. (n.d.). https://clinicaltrials.gov/ct2/show/study/NCT02181075?term= Thermodox\&rank $=5$ (accessed January 12,2017 ).

[38] G. Dimcevski, et al., A human clinical trial using ultrasound and microbubbles to enhance gemcitabine treatment of inoperable pancreatic cancer, J. Control. Release 243 (2016) 172-181.

[39] Blood-brain barrier disruption using transcranial MRI-guided focused ultrasound Clin. Trials Gov. (n.d.). https://clinicaltrials.gov/ct2/show/study/NCT02343991? term $=$ NCT02343991\&rank $=1$ (accessed January 12, 2017).

[40] F. Cavalieri, M. Zhou, M. Tortora, B. Lucilla, M. Ashokkumar, Methods of preparation of multifunctional microbubbles and their in vitro/in vivo assessment of stability, functional and structural properties, Curr. Pharm. Des. 18 (15) (2012) 2135-2151.

[41] S.R. Sirsi, M.A. Borden, Microbubble compositions, properties and biomedical applications, Bubble Sci. Eng. Tech. 1 (1-2) (2009) 3-17.

[42] P. Koczera, et al., Fluorescently labeled microbubbles for facilitating translational molecular ultrasound studies, Drug Deliv. Transl. Res. 2 (1) (2012) 56-64.
[43] S. Joseph, C. Olbrich, J. Kirsch, M. Hasbach, A. Briel, M. Schirner, A real-time in vitro assay for studying functional characteristics of target-specific ultrasound contrast agents, Pharm. Res. 21 (6) (2004) 920-926.

[44] M. Palmowski, et al., Pharmacodynamics of streptavidin-coated cyanoacrylate microbubbles designed for molecular ultrasound imaging, Investig. Radiol. 43 (3) (2008) 162-169.

[45] C. Olbrich, et al., The in vitro stability of air-filled polybutylcyanoacrylate microparticles, Biomaterials 27 (19) (2006) 3549-3559.

[46] S. Fokong, et al., Ultrasound molecular imaging of E-selectin in tumor vessels using poly n-butyl cyanoacrylate microbubbles covalently coupled to a short targeting peptide, Investig. Radiol. 48 (12) (2013) 843-850.

[47] A. Curaj, et al., Noninvasive molecular ultrasound monitoring of vessel healing after intravascular surgical procedures in a preclinical setup, Arterioscler. Thromb. Vasc. Biol. 35 (6) (2015) 1366-1373.

[48] $\mathrm{Z}$. Wu et al, Rhodamine-loaded intercellular adhesion molecule-1-targeted microbubbles for dual-modality imaging under controlled shear stresses, Circ. Cardiovasc. Imaging 6 (6) (2013) 974-981.

[49] A. Rix, et al., Molecular ultrasound imaging of $\alpha_{\mathrm{v}} \beta_{3}$-integrin expression in carotid arteries of pigs after vessel injury, Investig. Radiol. 51 (12) (2016) 767-775.

[50] R.A. Linker, et al., In vivo molecular imaging of adhesion molecules in experimental autoimmune encephalomyelitis (EAE), J. Autoimmun. 25 (3) (2005) 199-205.

[51] M. Reinhardt, et al., Ultrasound derived imaging and quantification of cell adhesion molecules in experimental autoimmune encephalomyelitis (EAE) by Sensitive Particle Acoustic Quantification (SPAQ), Neurolmage 27 (2) (2005) 267-278.

[52] I. Spivak, et al., Low-dose molecular ultrasound imaging with E-selectin-targeted PBCA microbubbles, Mol. Imaging Biol. 18 (2) (2016) 180-190.

[53] S. Fokong, et al., Image-guided, targeted and triggered drug delivery to tumors using polymer-based microbubbles, J. Control. Release 163 (1) (2012) 75-81.

[54] M. Palmowski, et al., Molecular profiling of angiogenesis with targeted ultrasound imaging: early assessment of antiangiogenic therapy effects, Mol. Cancer Ther. 7 (1) (2008) 101-109.

[55] M. Palmowski, et al., Molecular ultrasound imaging of early vascular response in prostate tumors irradiated with carbon ions, Neoplasia 11 (9) (2009) 856-863.

[56] BR55 in prostate cancer: an exploratory clinical trial, Clin. Trials Gov. (n.d.). https:// clinicaltrials.gov/ct2/show/NCT01253213 (accessed January 12, 2017).

[57] A pilot trial using BR55 ultrasound contrast agent in the assessment of prostate cancer, Clin. Trials Gov. (n.d.). https://clinicaltrials.gov/ct2/show/NCT02142608?term = NCT02142608\&rank=1 (accessed January 12, 2017).

[58] T. Lammers, et al., Theranostic USPIO-loaded microbubbles for mediating and monitoring blood-brain barrier permeation, Adv. Funct. Mater. 25 (1) (2015) 36-43.

[59] B. Theek, et al., Sonoporation enhances liposome accumulation and penetration in tumors with low EPR, J. Control. Release 231 (2016) 77-85.

[60] J.R. Eisenbrey, M.C. Soulen, M.A. Wheatley, Delivery of encapsulated doxorubicin by ultrasound-mediated size reduction of drug-loaded polymer contrast agents, IEEE Trans. Biomed. Eng. 57 (1) (2010) 24-28.

[61] M.C. Cochran, et al., Disposition of ultrasound sensitive polymeric drug carrier in a rat hepatocellular carcinoma model, Acad. Radiol. 18 (11) (2011) 1341-1348.

[62] M.C. Cochran, J. Eisenbrey, R.O. Ouma, M. Soulen, M.A. Wheatley, Doxorubicin and paclitaxel loaded microbubbles for ultrasound triggered drug delivery, Int. J. Pharm. 414 (1-2) (2011) 161-170.

[63] A.K.O. Aslund, et al., Nanoparticle delivery to the brain - by focused ultrasound and self-assembled nanoparticle-stabilized microbubbles, J. Control. Release 220 (Pt A) (2015) 287-294

[64] S. Eggen, et al., Ultrasound-enhanced drug delivery in prostate cancer xenografts by nanoparticles stabilizing microbubbles, J. Control. Release 187 (2014) 39-49. 